\title{
ON THE NONLINEAR TRANSVERSE VIBRATIONS AND STABILITY OF AN AXIALLY ACCELERATING BEAM
}

\author{
H. R. ÖZ \\ Department of Mechanical Engineering,Celal Bayar University, \\ 45140 Muradiye, Manisa, Turkey
}

\begin{abstract}
Nonlinear vibrations and stability analysis of an axially moving EulerBernoulli type beam are investigated. The beam is on fixed supports and moving with a harmonically varying velocity about a constant mean value. The method of multiple scales is used in the analysis. Nonlinear frequencies depending on vibration amplitudes are obtained. Stability and bifurcations of steady-state solutions are analyzed for frequencies close to two times any natural frequency. It is shown that the amplitudes are bounded in time for frequencies close to zero. The effect of fixed supports is discussed.
\end{abstract}

\section{INTRODUCTION}

Due to their technological importance the vibrations of band-saws, fiber textiles, magnetic tapes, paper sheets, aerial tramways, pipes transporting fluids, threadlines, belts were investigated by many researchers. These problems are investigated by using either a string model or a beam model. For the linear model review of the literature were performed by Ulsoy et al. [1] and Wickert and Mote [2].

It was shown that nonlinear effects in string vibrations became more significant at velocities close to critical speed [3]. Ames et al. [4] considered a harmonically excited traveling string using finite difference method. Wickert [5] analyzed free nonlinear vibrations by including stretching effects for a moving beam over the sub- and supercritical speed ranges. Moon and Wickert [6] studied nonlinear vibration of a prototypical power transmission belt system, which was excited by pulleys having slight eccentricity through experimental and analytical methods. Chakraborty et al. [7] investigated the free and forced vibrations for a traveling beam. Öz et al. [8] considered a variable speed nonlinear beam model resting on simple supports and obtained nonlinear frequencies depending on the amplitude. Frequency response curves and stability of the solutions were investigated. Nonlinear vibrations of a tensioned pipe conveying fluid and traveling beam with variable velocity were investigated [9-11] and the same results were found in agreement with reference [8].

In this study, an Euler-Bernoulli type beam model is considered. The fixed ends introduce nonlinear effects due to stretching. Approximate solutions are obtained using the 
method of multiple scales. The transport velocity is assumed to vary harmonically about a constant mean value. The natural frequencies depending on mean velocity are plotted for the first two modes and for different flexural stiffness values. Fixed supports increase natural frequencies compared with simple supports [8]. Three distinct cases are considered depending on the frequency of velocity fluctuations:

i) Velocity fluctuation frequency away from zero or two times one of the natural frequencies.

ii) Velocity fluctuation frequency close to zero

iii) Velocity fluctuation frequency close to two times one of the natural frequencies.

For the first case, the amplitudes of vibration are found to be constant and the phases vary linearly in time. The nonlinear frequencies are calculated. For the second case, it is shown that the amplitudes are bounded in time. For the last case, the frequency-response curves are plotted. Two non-trivial steady-state solutions bifurcate from the trivial steady-state solution. Stability of these solutions is investigated. The effects of fixed boundary conditions are discussed and compared with simply supported beam.

\section{EQUATION OF MOTION}

The dimensionless equation of motion is [8],

$\ddot{u}+2 v \dot{u}^{\prime}+\dot{v} u^{\prime}+\left(v^{2}-1\right) u^{\prime \prime}+v_{f}^{2} u^{i v}=\frac{1}{2} \varepsilon v_{1}^{2} u^{\prime \prime} \int_{0}^{1} u^{\prime 2} d x$

$u$ denotes the transverse displacement, dot denotes derivative with respect to time $(t)$ and prime denotes derivative with respect to the spatial derivative $(\mathrm{x}), \varepsilon$ is a small parameter $(\varepsilon<<1), v_{f}^{2}$ and $v_{1}^{2}$ denote flexural and longitudinal stiffnesses respectively. Equation (1) represents a weakly nonlinear system. The boundary conditions for fixed-fixed beam are

$u(0, t)=0, u(1, t)=0, u^{\prime}(0, t)=0, u^{\prime}(1, t)=0$

Assuming small fluctuations about a constant mean velocity, one writes

$v=v_{0}+\varepsilon v_{1} \sin \Omega t$

where $\varepsilon v_{1}$ and $\Omega$ are the amplitude and the frequency of fluctuations respectively. Although the amplitude of fluctuations and nonlinear effects seem to be connected through the parameter $\varepsilon$, their independency is assured by inclusion of an arbitrary parameter $v_{1}$ of order one. In the next section, the approximate solution of the equation of motion will be given directly from reference [8]. 


\section{PRINCIPAL PARAMETRIC RESONANCES}

In the direct method, one assumes an expansion of the form

$\mathrm{u}(\mathrm{x}, \mathrm{t} ; \varepsilon)=\mathrm{u}_{0}\left(\mathrm{x}, \mathrm{T}_{n}, \mathrm{~T}_{1}\right)+\varepsilon \mathrm{u}_{1}\left(\mathrm{x}, \mathrm{T}_{0}, \mathrm{~T}_{1}\right)+\ldots$

where $1_{0}=\mathrm{t}$ and $\mathrm{l}_{1}=\mathrm{zt}$. Time derivatives are defined as

$\frac{d}{d t}=D_{0}+\varepsilon D_{1}+\ldots, \frac{d^{2}}{d t^{2}}=D_{0}^{2}+2 \varepsilon D_{0} D_{1}+\ldots$

where $D_{i}=\partial / \partial T_{i}$. Applying the method of multiple scales [8-11], at order 1 the solution is

$$
u_{0}=A_{n}(T) e^{i \omega_{n} T_{0}} \psi_{n}(x)+\bar{A}_{n}\left(T_{1}\right) e^{-i \omega_{n} T_{0}} \bar{\psi}_{n}(x)
$$

where $\omega_{n}$ represent the natural frequencies and $A_{n}$ is complex amplitude, $\bar{A}_{n}$ is complex conjugate of the amplitude. Following a similar calculation in [8-11] the shape functions are obtained as

$$
\begin{aligned}
& \psi_{n}(x)=c_{1}\left\{e^{i \beta_{1 n} x}-\frac{\left(\beta_{4 n}-\beta_{1 n}\right)\left(e^{i \beta_{3 n}}-e^{i \beta_{1 n}}\right)}{\left(\beta_{4 n}-\beta_{2 n}\right)\left(e^{i \beta_{3 n}}-e^{i \beta_{2 n}}\right)} e^{i \beta_{2 n} x}-\frac{\left(\beta_{4 n}-\beta_{1 n}\right)\left(e^{i \beta_{2 n}}-e^{i \beta_{1 n}}\right)}{\left(\beta_{4 n}-\beta_{3 n}\right)\left(e^{i \beta_{2 n}}-e^{i \beta_{3 n}}\right)} e^{i \beta_{3 n} x}\right. \\
&+\left[-1+\frac{\left(\beta_{4 n}-\beta_{1 n}\right)\left(e^{i \beta_{3 n}}-e^{i \beta_{1 n}}\right)}{\left(\beta_{4 n}-\beta_{2 n}\right)\left(e^{i \beta_{3 n}}-e^{i \beta_{2 n}}\right)}+\frac{\left(\beta_{4 n}-\beta_{1 n}\right)\left(e^{i \beta_{2 n}}-e^{i \beta_{1 n}}\right)}{\left.\left.\left(\beta_{4 n}-\beta_{3 n}\right)\left(e^{i \beta_{2 n}}-e^{i \beta_{3 n}}\right)\right] e^{i \beta_{4 n} x}\right\}}\right.
\end{aligned}
$$

where $\beta_{\text {in }}$ are eigenvalues. The velocity dependent natural frequencies are given in Figures 1 and 2 for the first and second modes respectively for different flexural stiffness values . The frequencies decrease with increasing mean velocity. As the flexural rigidity increases $\left(v_{f}\right)$, the frequencies increase. Solutions depend on three distinct cases which will be investigated separately.

i) $\Omega$ away from $2 \omega_{\mathrm{n}}$ or 0

In this case, the solvability condition [8-12] is obtained as

$D_{1} A_{n}-k_{3} A_{n}^{2} \bar{A}_{n}=0$

where $\mathrm{k}_{3}$ is defined to be 
$\mathrm{k}_{3}=\mathrm{v}_{1}^{2} \frac{\frac{1}{2} \int_{0}^{1} \bar{\psi}_{\mathrm{n}}^{\prime \prime} \bar{\psi}_{\mathrm{n}} \mathrm{dx} \int_{0}^{1} \psi_{\mathrm{n}}^{\prime 2} \mathrm{dx}+\int_{0}^{1} \bar{\psi}_{\mathrm{n}} \psi_{\mathrm{n}}^{\prime \prime} \mathrm{dx} \int_{0}^{1} \psi_{\mathrm{n}}^{\prime} \bar{\psi}_{\mathrm{n}}^{\prime} \mathrm{dx}}{2\left(\mathrm{i} \omega_{\mathrm{n}} \int_{0}^{1} \bar{\psi}_{\mathrm{n}} \psi_{\mathrm{n}} \mathrm{dx}+v_{0} \int_{0}^{1} \bar{\psi}_{\mathrm{n}} \psi_{\mathrm{n}} \mathrm{dx}\right)}$

where $i$ is the complex number and $\omega_{n}$ is the natural frequency.

ii) $\Omega$ close to 0

The nearness of fluctuation frequency to zero can be expressed as

$\Omega=\varepsilon \sigma$

where $\sigma$ is a detuning parameter. For this case, the solvability condition becomes

$\mathrm{D}_{1} \mathrm{~A}_{\mathrm{n}}+\left(\mathrm{k}_{1} \cos \sigma \mathrm{T}_{1}+\mathrm{k}_{2} \sin \sigma \mathrm{T}_{1}\right) \mathrm{A}_{\mathrm{n}}-\mathrm{k}_{3} \mathrm{~A}_{\mathrm{n}}^{2} \overline{\mathrm{A}}_{\mathrm{n}}=0$

where

$$
\begin{aligned}
& \mathrm{k}_{1}=\frac{v_{1} \Omega \int_{0}^{1} \bar{\psi}_{\mathrm{n}} \psi_{\mathrm{n}} \mathrm{dx}}{2\left(\mathrm{i} \omega_{\mathrm{n}} \int_{0}^{1} \bar{\psi}_{\mathrm{n}} \psi_{\mathrm{n}} \mathrm{dx}+\mathrm{v}_{0} \int_{0}^{1} \bar{\psi}_{\mathrm{n}} \psi_{\mathrm{n}}^{\prime} \mathrm{dx}\right)} \\
& \mathrm{k}_{2}=\frac{\mathrm{v}_{0} v_{1} \int_{0}^{1} \bar{\psi}_{\mathrm{n}} \psi_{\mathrm{n}} \mathrm{dx}+\mathrm{i} v_{1} \omega_{\mathrm{n}} \int_{0}^{1} \psi_{\mathrm{n}}^{\prime} \bar{\psi}_{\mathrm{n}} \mathrm{dx}}{\mathrm{i} \omega_{\mathrm{n}} \int_{0}^{1} \psi_{\mathrm{n}} \bar{\psi}_{\mathrm{n}} \mathrm{dx}+\mathrm{v}_{0} \int_{0}^{1} \bar{\psi}_{\mathrm{n}} \psi_{\mathrm{n}}^{\prime} \mathrm{dx}}
\end{aligned}
$$

iii) $\Omega$ close to $2 \omega_{\mathrm{n}}$

For this case, one writes

$\Omega=2 \omega_{n}+\varepsilon \sigma$

The solvability condition $[8]$ is

$$
D_{1} A_{n}+k_{0} \bar{A}_{n} e^{i \sigma T_{1}}-k_{3} A_{n}^{2} \bar{A}_{n}=0
$$

where 
$k_{0}=\frac{v_{1}\left(\frac{\Omega}{2}-\omega_{n}\right)_{0}^{l} \bar{\psi}_{n} \bar{\psi}_{n}^{\prime} d x-i v_{0} v_{1} \int_{0}^{1} \bar{\psi}_{n} \bar{\psi}_{n}^{\prime \prime} d x}{2\left(i \omega_{n} \int_{0}^{1} \psi_{n} \bar{\psi}_{n} d x+v_{0} \int_{0}^{1} \bar{\psi}_{n} \psi_{n}^{\prime} d x\right)}$

Amplitude-phase variation, steady-state solutions and their stability and bifurcation analysis will be discussed in the next section.

\section{STABILITY AND BIFURCATIONS}

The three different cases will be considered in this section.

i) $\Omega$ away from 0 or $2 \omega_{\mathrm{n}}$

Following similar calculations in [8-11], and expressing the complex amplitudes $A_{n}$ in polar form

$$
A_{n}=\frac{1}{2} a_{n} e^{i \beta_{n}}
$$

the nonlinear frequency can be obtained as

$$
\left(\omega_{n}\right)_{n !}=\omega_{n}+\varepsilon \frac{1}{4} k_{31} a_{0 n}^{2}
$$

where $\mathrm{a}_{0}$ is constant real amplitude, $\mathrm{k}_{31}$ is imaginary part of $\mathrm{k}_{3}$ and $\mathrm{n}$ denotes the modes. Nonlinear frequency versus amplitude are drawn in Figures 3 and 4 for the first and second modes respectively. When the mean velocity is closer to the critical velocity where divergence instability occurs, the nonlinear effects are more significant. The nonlinear frequency values shift to higher values for fixed end conditions compared with simply supported traveling beam in [8].

\section{ii) $\Omega$ close to 0}

For this case, the amplitude equation is $[8,9]$

$a_{n}=a_{O n} e^{\frac{1}{\sigma}\left(-k_{1 R} \sin \sigma T_{1}+k_{2 R} \cos \sigma T_{1}\right)}$

where $k_{1 R}$ and $k_{2 R}$ are real parts of $k_{1}$ and $k_{2}$ respectively. Since $-1 \leq \sin \sigma T_{1} \leq 1$ and $-1 \leq \cos \sigma \mathrm{T}_{1} \leq 1$, the amplitudes are bounded in time. In addition to the nontrivial solution, the trivial solution $a_{n}=0$ also exists. 
iii) $\Omega$ close to $2 \omega_{\mathrm{n}}$

Following derivations in $[8,9]$ one gets $a_{n}{ }^{\prime}=\beta_{n}{ }^{\prime}=0$ (amplitude and phase) for steady-state solutions, so that the trivial solution $a_{n}=0$ is a solution. Two non-trivial solutions can be found as

$$
\begin{aligned}
& \sigma_{1}=\frac{1}{2} \mathrm{k}_{3 \mathrm{I}} \mathrm{a}_{\mathrm{n}}^{2}-2 \sqrt{\mathrm{k}_{0 \mathrm{I}}^{2}+\mathrm{k}_{0 \mathrm{R}}^{2}} \\
& \sigma_{2}=\frac{1}{2} \mathrm{k}_{3 \mathrm{I}} \mathrm{a}_{\mathrm{n}}^{2}+2 \sqrt{\mathrm{k}_{01}^{2}+\mathrm{k}_{0 \mathrm{R}}^{2}}
\end{aligned}
$$

where $\mathrm{k}_{\mathrm{OR}}$ and $\mathrm{k}_{\mathrm{OI}}$ are real and imaginary parts of $\mathrm{k}_{0}$ respectively. In the frequency-response diagram, these two nontrivial solutions bifurcate from the trivial solution. For a given frequency detuning value $(\sigma)$, the trivial and nontrivial solutions may coexist and determination of the stability of these steady-state solutions become important. The trivial solution is stable when

$$
\begin{aligned}
& \sigma>2 \sqrt{\mathrm{k}_{0 \mathrm{R}}^{2}+\mathrm{k}_{0 \mathrm{I}}^{2}} \\
& \sigma<-2 \sqrt{\mathrm{k}_{0 \mathrm{R}}^{2}+\mathrm{k}_{0 \mathrm{I}}^{2}}
\end{aligned}
$$

and unstable otherwise.

Performing analysis for the stability of nontrivial solutions one finds $\sigma_{1}$ curve is always stable and $\sigma_{2}$ curve is always unstable [8,9].

In Figure 5, a mean speed much lower than the critical speed and in Figure 6, a mean speed close to the critical speed are chosen. Solid lines show stable solutions and dashed lines show unstable solutions. There are two supercritical pitchfork bifurcation points indicated as $\sigma_{1}$ and $\sigma_{2}$ in the figures. If a frequency of $\sigma<\sigma_{1}$ is chosen, only trivial solution exists and is stable. If frequency is increased, after the $\sigma=\sigma_{1}$ bifurcation point, the trivial solution looses stability and a nontrivial response is obtained. The trivial solution is stable after the second bifurcation point $\sigma=\sigma_{2}$. The nontrivial solution starting from $\sigma_{2}$ is not stable. The nonlinear effects are more significant for speeds close to the critical speed. For fixed end conditions the stability curves shift to higher forcing values compared with [8]. 


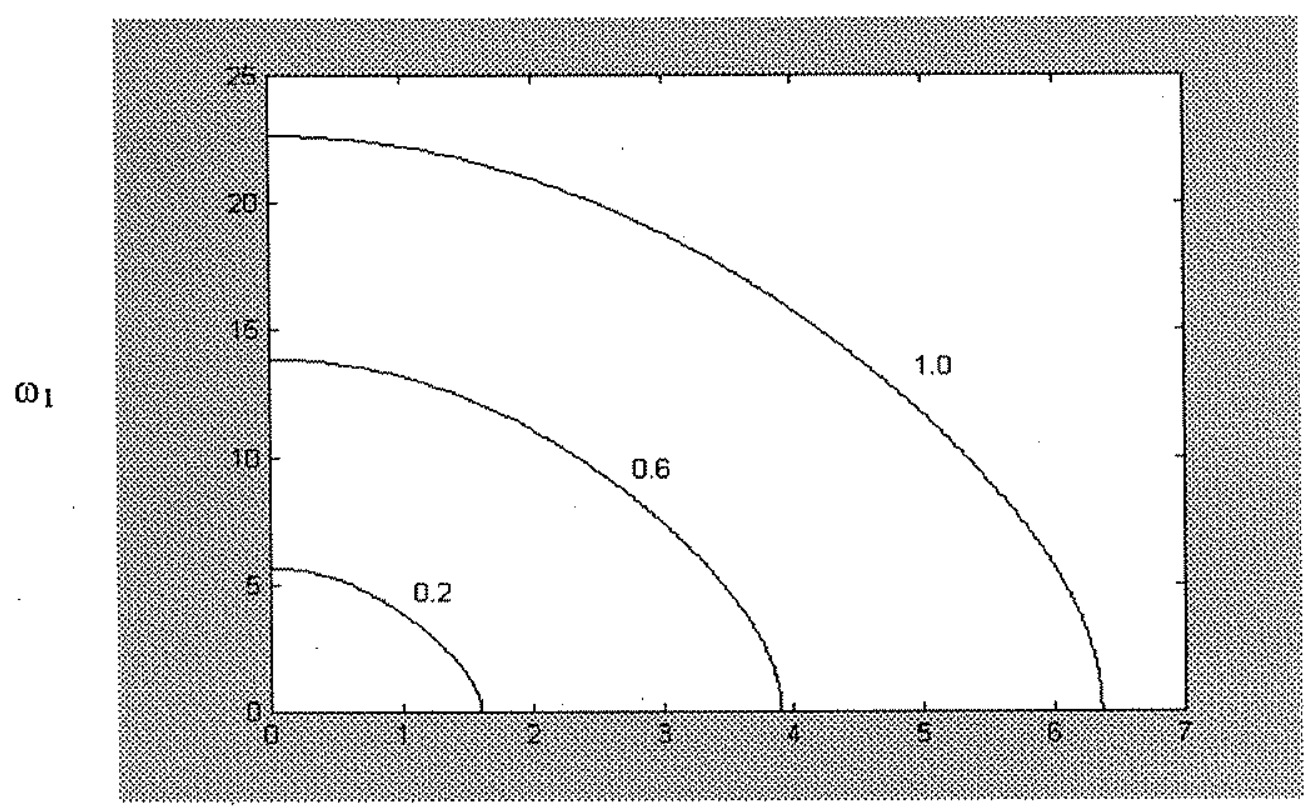

$\mathrm{v}_{0}$

Figure 1. Variation of natural frequency with mean velocity for different flexural stiffness values for the first mode ( $v_{f}$ values are indicated on the curves).

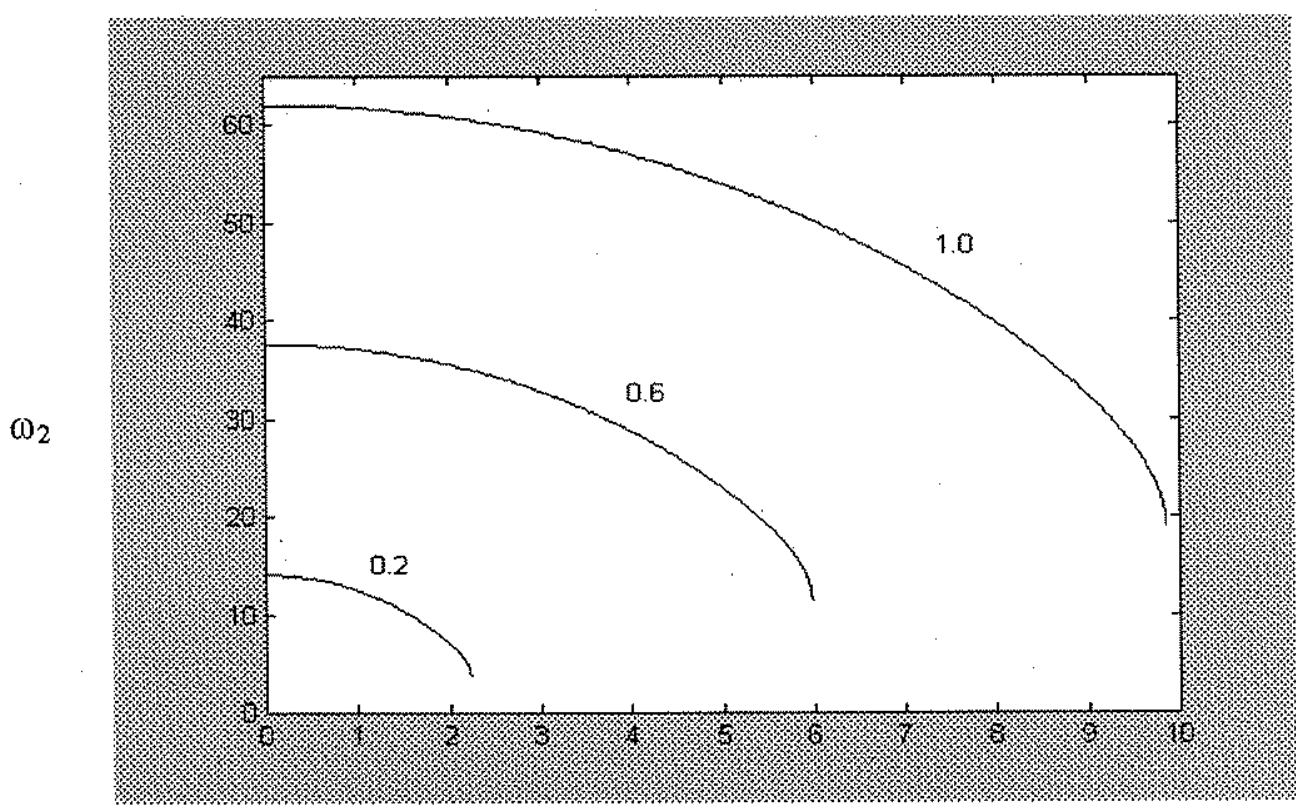

$v_{0}$

Figure 2. Variation of natural frequency with mean velocity for different flexural stiffness values for the second mode ( $v_{f}$ values are indicated on the curves). 


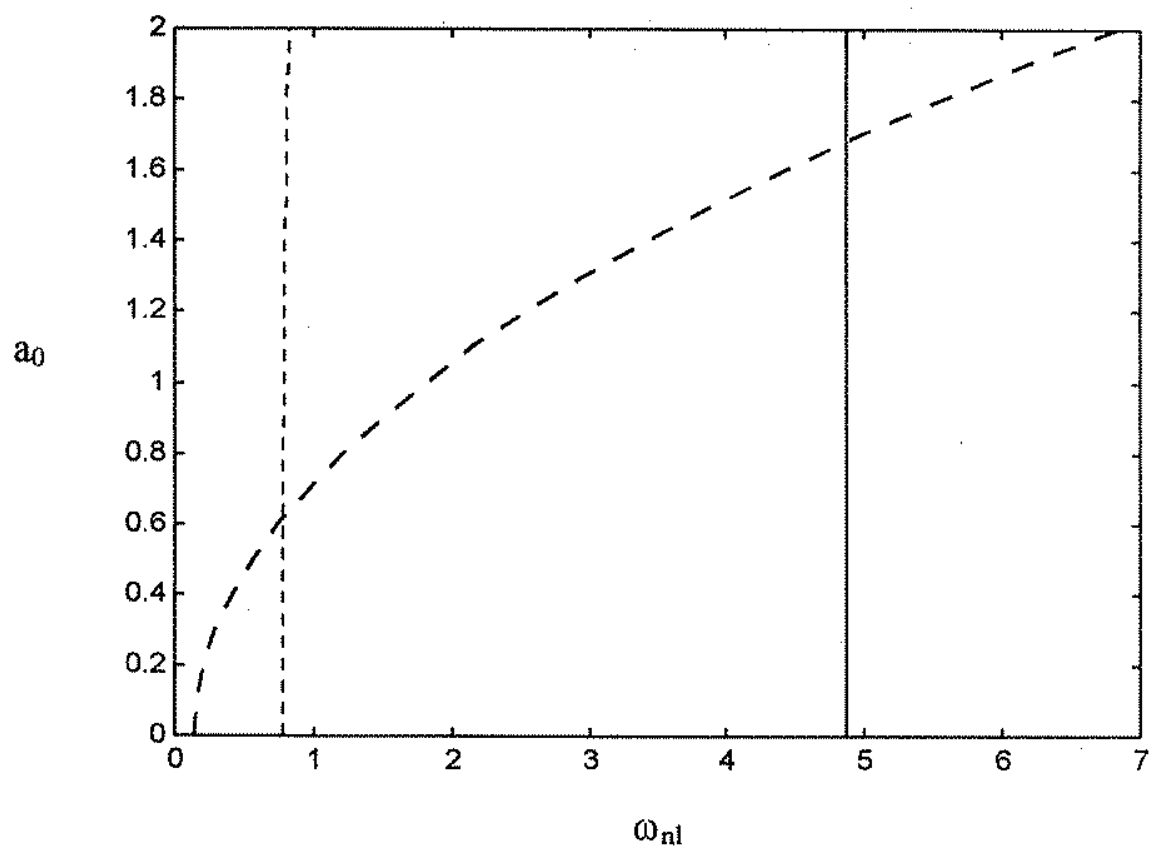

Figure 3. Variation of nonlinear natural frequencies with amplitudes for different mean velocities for the first mode $v_{f}=1.0,\left(v_{0}=6.090-1 v_{0}=6.355 \ldots\right),\left(v_{0}=6.362 \ldots\right)$. Critical velocity is $v_{\mathrm{cr}}=6.36236$.

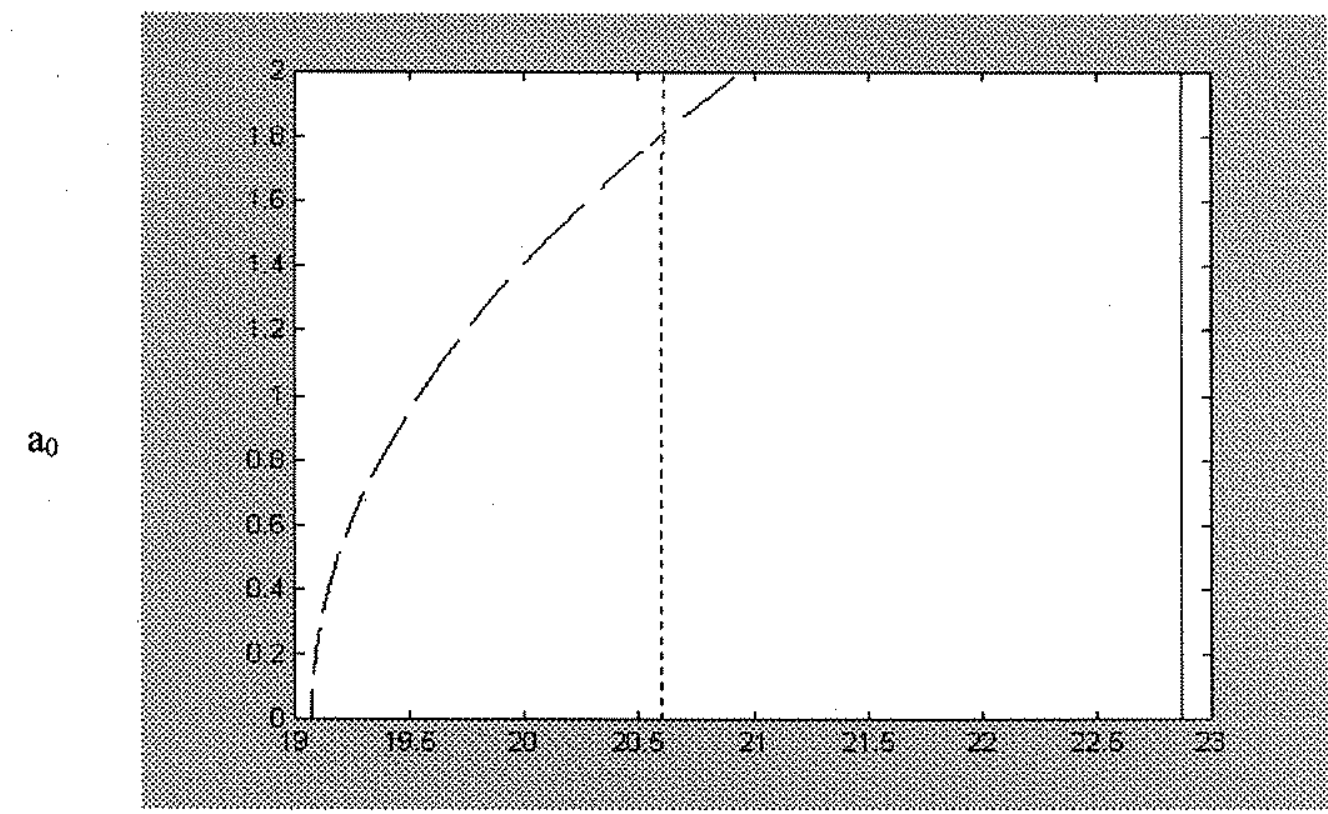

$\omega_{\mathrm{nl}}$

Figure 4. Variation of nonlinear natural frequencies with amplitudes for different mean velocities for the second mode $v_{f}=1.0,\left(v_{0}=9.760-\longrightarrow\right),\left(v_{0}=9.830 \ldots\right),\left(v_{0}=9.847 \ldots\right)$. Critical velocity is $v_{\mathrm{cr}}=9.8473$. 


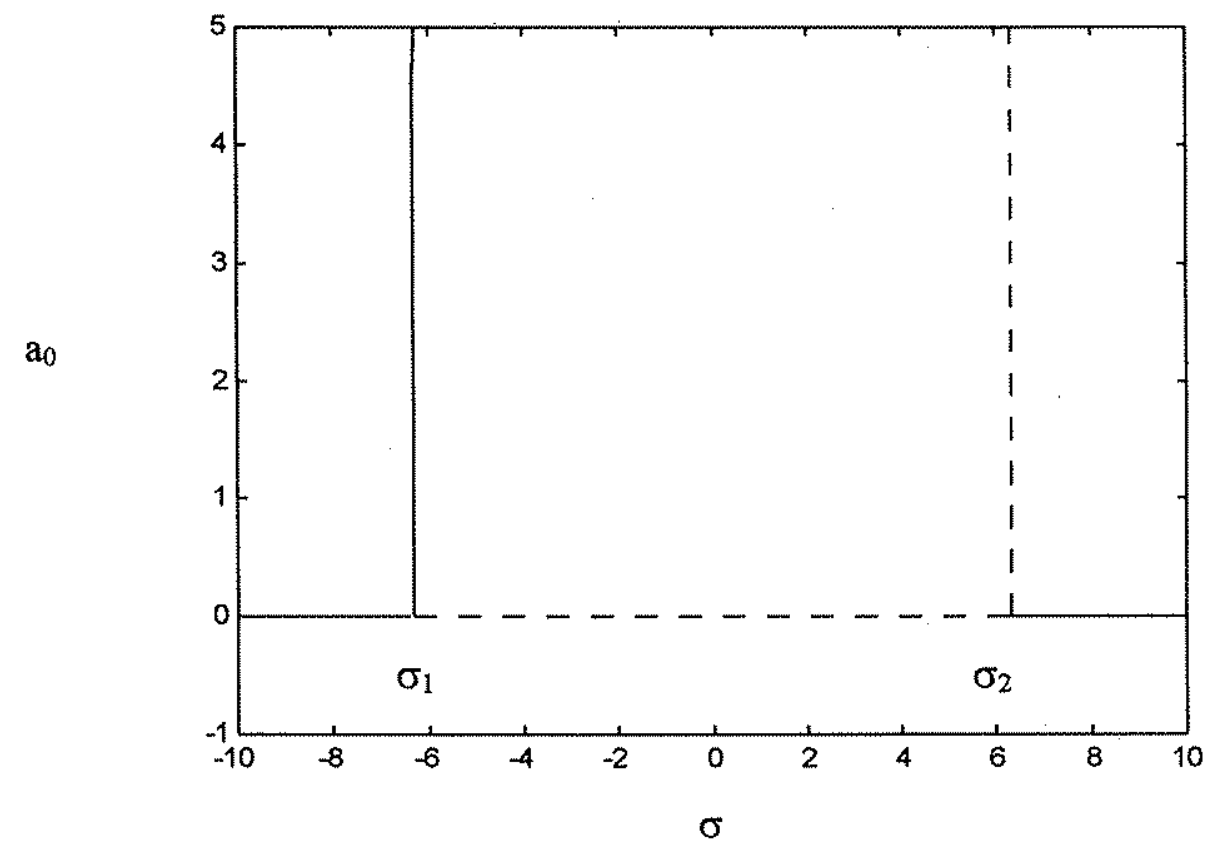

Figure 5. Frequency-response curves for a velocity much lower than the critical velocity for the first mode $\left(\mathrm{v}_{\mathrm{f}}=1.0, \mathrm{v}_{0}=5.9\right.$ Solid lines and dashed lines denote stable and unstable solutions respectively).

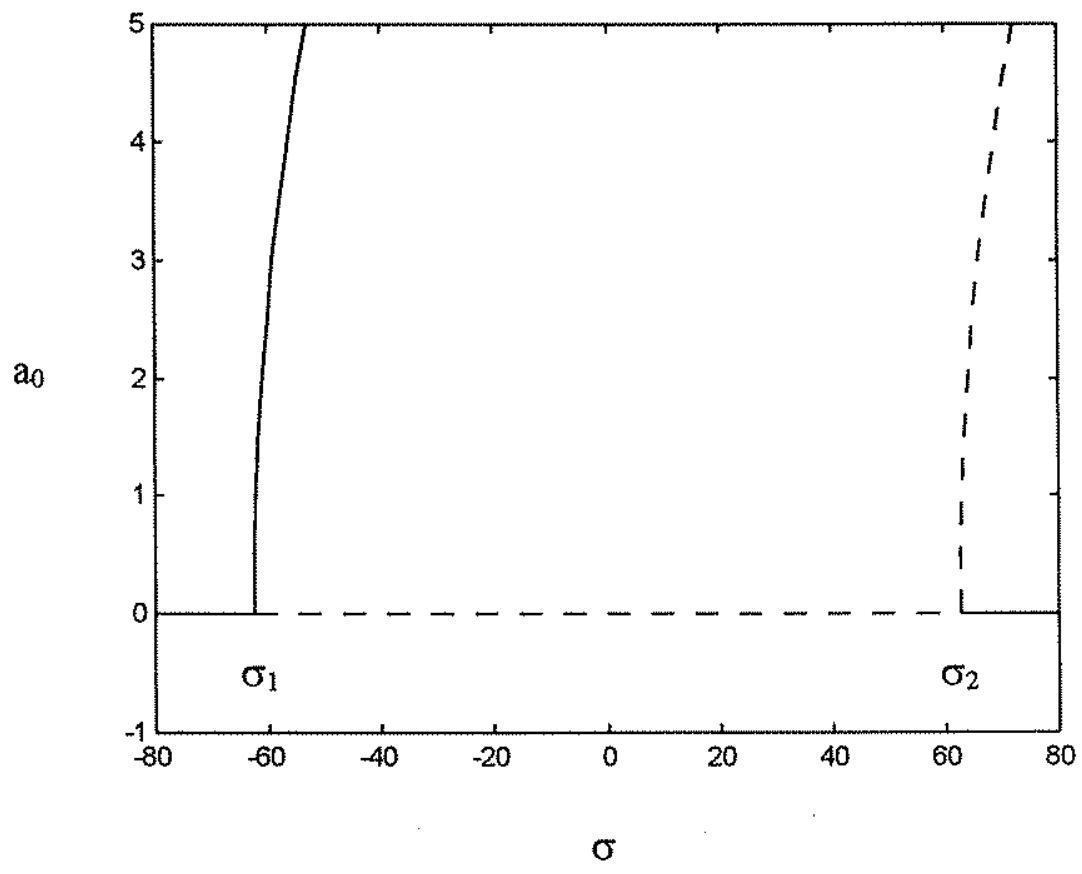

Figure 6. Frequency-response curves for a velocity close to the critical velocity for the first mode $\left(v_{\mathrm{f}}=1.0, v_{0}=6.357\right.$ Solid lines and dashed lines denote stable and unstable solutions respectively). 


\section{CONCLUDING REMARKS}

The nonlinear transverse vibrations of an axially accelerating Euler-Bernoulli beam are analyzed and the stability analysis is performed. The beam is on fixed supports which introduce nonlinearity due to stretching of neutral axis. The axial velocity is assumed to vary harmonically about a constant mean value with small fluctuation amplitudes. The method of multiple scales is applied to the equation of motion. The natural frequencies decrease with increasing mean speed and divergence instability occurs at the critical speed. The natural frequencies for fixed-fixed traveling beam are greater than those of simply supported beam and so are critical speeds. For the frequencies away from zero or two times the natural frequency, the amplitudes of vibration are constant and the phases vary linearly in time. The nonlinear frequencies depending on the amplitudes are obtained. The nonlinear effects become important for speeds close to critical speed. When fluctuation frequencies are close to zero, it is shown that the amplitudes are bounded in time. When fluctuation frequencies are close to two times the natural frequency, stability and bifurcations of steady-state solutions are analyzed and the frequency-response curves are plotted. Two non-trivial steady-state solutions bifurcate from the trivial steady-state solution. There are two supercritical pitchfork bifurcation points. After the first bifurcation point, the trivial solution looses stability and a nontrivial response is acquired. The reverse is true for the second bifurcation point. The fixed supports enlarges unstable region, shift stable curve to lower frequency detuning values and unstable curve to higher values.

Acknowledgements- This work is supported by the Scientific and Technical Research Council of Turkey under project no. MISAG-119.

\section{REFERENCES}

1. A. G. Ulsoy, C. D. Mote JR., and R. Syzmani, Principal developments in band saw vibration and stability research, Holz als Roh- und Werkstoff 36, 273-280, 1978.

2. J. A. Wickert and C. D. Mote JR, Current research on the vibration and stability of axially moving materials, Shock and Vibration Digest 20(5), 3-13, 1988.

3. C. D. Mote JR, On the non-linear oscillation of an axially moving string, Journal of Applied Mechanics 33, 463-464, 1966.

4. W. F. Ames, S. Y. Lee and J. N. Zaiser, Non-linear vibration of a traveling threadline, International Journal of Non-Linear Mechanics 3, 449-469, 1968.

5. J. A. Wickert, Non-linear vibration of a traveling tensioned beam, International Journal of Non-Linear Mechanics 27, 503-517, 1992.

6. J. Moon and J. A. Wickert, Non-linear vibration of power transmission belts, Journal of Sound and Vibration 200(4), 419-431, 1997.

7. G. Chakraborty, A. K. Mallik and H. Hatwal, Non-linear vibration of a traveling beam, International Journal of Non-Linear Mechanics 34, 655-670, 1999.

8. H. R. Öz, M. Pakdemirli and H. Boyac1, Nonlinear vibrations and stability of an axially moving beam with time dependent velocity, International Journal of Non-Linear Mechanics, 36(1), 107-115, 2001. 
9. H. R. Öz, Non-linear vibrations and stability analysis of tensioned pipes conveying fluid with variable velocity, International Journal of Non-Linear Mechanics, (to be published).

10. H.R. ÖZ, M. PAKDEMIRLI, "Vibrations of an axially moving beam with time dependent velocity", Journal of Sound and Vibration, 227(2), 239-257, 1999.

11. H. R. ÖZ, "On the vibrations of an axially traveling beam on fixed supports with variable velocity" Journal of Sound and Vibration (to be published).

12. A. H. Nayfeh, Introduction to Perturbation Techniques, John Wiley, New York, 1981.

13. A. H. Nayfeh and D. T. Mook, Nonlinear Oscillations, John Wiley, New York, 1979.

14. A. H. Nayfeh and B. Balachandran, Applied Nonlinear Dynamics, Wiley-Interscience, New York, 1995. 